\title{
Calidad de imagen y reducción de dosis en angiotomografía computarizada de arterias coronarias usando protocolo de baja energía
}

\author{
Felipe Allende $\mathrm{N}^{\prime}$, Karina Araya $\mathrm{V}^{2}$, Esteban Madariaga $\mathbf{M}^{3}$, Patricia Bitar $\mathrm{H}^{4}$, Paola Paolinelli $\mathrm{G}^{4}$.
}

1. Tecnólogo Médico, Magister en Ciencias de la Educación. Universidad Mayor. Santiago, Chile.

2. Licenciada en Tecnología Médica, Hospital Clínico Universidad Católica. Santiago, Chile.

3. Licenciado en Tecnología Médica, Clínica Tabancura. Santiago, Chile.

4. Médico Radiólogo, Clínica Las Condes. Santiago, Chile.

\section{Coronary artery CT image quality and dose reduction using low energy protocol}

\begin{abstract}
The objective of this study was to quantify the radiation dose reduction without impairing image quality, using 100 and 120 kiloVolts acquisition protocols. We analyzed 37 non-obese patients in a private hospital in Santiago, Chile, of which 20 were obtained with a protocol of 120 kiloVolts and the remaining 17 with low energy protocol. The quality of the image was evaluated quantitatively and qualitatively. The average image quality score for the 120 and 100 kiloVolts groups was $3.25 \pm 0.8$ and $3.41 \pm 0.7$ respectively, demonstrating an improvement in the image quality, although at a non-significant value $(p=0.58)$. In the images obtained with the 100 kiloVolts protocol, a reduction of $41.99 \%$ in the radiation dose was demonstrated in addition to a significant increase of the intensity of the signal and the noise. It was possible to demonstrate a decrease of the radiation dose without detriment in the diagnostic image quality in coronary angiography using computed tomography.
\end{abstract}

Key words: Computed tomography angiography, Computed tomography, Effective dose, Image quality.

Resumen. El objetivo de este estudio fue cuantificar la disminución de la dosis de radiación sin perjudicar la calidad de imagen, utilizando protocolos de adquisición de 100 y 120 kilovolts. Se analizaron 37 pacientes no obesos en un hospital privado de Santiago de Chile, de los cuales 20 fueron obtenidos con un protocolo de 120 kilovolts y los 17 restantes con protocolo de baja energía. Se evaluó la calidad de imagen cuantitativa y cualitativamente. El promedio de puntuación de la calidad de imagen para los grupos de 120 y 100 kilovolts fue de 3,25 0,8 y 3,41 $\pm 0,7$ respectivamente, demostrando una mejora en la calidad de imagen, aunque en un valor no significativo $(p=0,58)$. En las imágenes obtenidas con el protocolo de 100 kilovolts, se demostró una reducción en la dosis de radiación de un 41,99\% además de un aumento significativo de la intensidad de la señal y del ruido. Se logró demostrar una disminución de la dosis de radiación sin perjuicio en la calidad de imagen diagnóstica en angiografía coronaria por tomografía computarizada.

Palabras clave: Angiografía por Tomografía computarizada, Tomografía computarizada, Calidad de imagen, Dosis efectiva.

Allende $F$, et al. Calidad de imagen y reducción de dosis en angiotomografía computarizada de arterias coronarias usando protocolo de baja energía. Rev Chil Radiol 2017; 23(3): 130-139.

Correspondencia: Felipe Allende Núñez / felipe.allende@umayor.cl

Trabajo recibido 28 de agosto de 2017. Aceptado para publicar el 25 de septiembre de 2017.

\section{Introducción}

El diagnóstico no invasivo de la enfermedad coronaria se ha instalado como una herramienta de gran utilidad en el contexto global de la patología cardiovascular, entidad que registró 17,5 millones de muertes en el año 2012 y de éstas, 7.4 millones a causa de cardiopatía isquémica, considerándose la primera causa de muerte a nivel mundial ${ }^{1}$. En Chile, 25.744 defunciones fueron asociadas al sistema circulatorio según los indicadores de salud del año 2013 , con una tasa de 149,25 por 100.000 habitantes, correspondiente a un $25,88 \%$ del total de defunciones de este mismo año, ocupando el primer lugar en las causas de muerte ${ }^{2}$. 
La angiotomografía computarizada (AngioTC) de arterias coronarias es una modalidad de imagen no invasiva para el estudio de las patologías cardiovasculares, que permite evaluar la pared, el lumen y la presencia de calcio en las arterias coronarias sin la necesidad de tener que recurrir a un cateterismo arterial. Permite valorar no solo la anatomía, sino también variantes anatómicas, anomalías, origen y trayecto de las arterias coronarias, además de la cuantificación directa de la estenosis, la evaluación de bypass, la valoración de la función cardiaca a través de imágenes volumétricas en ambas fases del ciclo cardiaco pudiendo determinar anomalías en la contractilidad miocárdica, grosor del miocardio y fracción de eyección. Sumado a lo anterior, esta técnica entrega información sobre las dimensiones auriculares y ventriculares, la función sistólica global y segmentaria del ventrículo izquierdo, características anatómicas y funcionales del ventrículo derecho, pericardio, septum interventricular e interauricular, así como permitir el estudio de la ubicación de las venas pulmonares y del seno coronario. Para esto son necesarias una alta resolución espacial y temporal, así como una excelente resolución de contraste ${ }^{3}$. La desventaja de este examen es la cantidad de dosis de radiación que los pacientes reciben por cada exploración, que se puede elevar a valores de $39 \mathrm{mSv}$, por este motivo se hace importante la optimización de dosis en dicho procedimiento 4 . La AngioTC de arterias coronarias tiene una sensibilidad de un $83 \%$, especificidad de $64 \%$, valor predictivo positivo de $99 \%$ y un valor predictivo negativo de $95 \%$ en pacientes sin enfermedad coronaria previa conocida ${ }^{5}$.

\section{Calidad de imagen}

En tomografía computarizada los principales factores que en forma interrelacionada determinan la calidad de la imagen son: El ruido, la resolución espacial y la resolución de contraste. La resolución espacial es la capacidad de diferenciar dos estructuras pequeñas y muy próximas como unidades independientes, es el detalle de la imagen, es decir, es una medida de lo pequeño que puede verse un objeto en una imagen, la que depende del tamaño de píxel y el grosor de corte, teniendo en cuenta que a menor tamaño de ambos parámetros se obtiene una mejor resolución espacial. La resolución de contraste es la capacidad de poder distinguir dos estructuras por su diferencia en la escala de grises, la cual está limitada por el contraste inherente y tamaño del objeto, su uniformidad y el ruido del equipo, una mayor resolución de contraste implica que los objetos más sutiles se puedan ver de forma rutinaria en la imagen. El ruido se define como la desviación estándar de una gran cantidad de pixeles que componen una imagen y se relaciona con la cantidad de señal recibida por los detectores, siendo que una mayor señal tiene como resultado una imagen con menor nivel de ruido y con esto la resolución contraste de la imagen aumenta ${ }^{6}$. Otro aspecto de la calidad de imagen específico de la AngioTC coronaria es la consideración del movimiento constante del corazón, que hace necesario tomar medidas para evitar la presencia de artefacto cinético, por lo que se debe controlar la frecuencia cardiaca del paciente al momento de realizar este examen. Para lograr una imagen de interpretación confiable, es necesario que el paciente presente una frecuencia cardiaca con un máximo de 65 latidos por minuto (Ipm), siendo necesaria la administración de beta bloqueadores por vía oral o intravenosa. También contribuyen a la obtención de una imagen libre de artefactos de movimiento la velocidad de adquisición que permiten los equipos con alto número de canales, la apnea del paciente y la adquisición en diástole ${ }^{7}$.

\section{Optimización de dosis de radiación en AngioTC coronaria}

Frente al aumento de los factores de riesgo para la enfermedad coronaria, las exploraciones por AngioTC coronaria han visto un importante aumento en el número de exámenes realizados teniendo una excelente aceptación clínica. La preocupación ante esto son los probables efectos estocásticos que se puedan producir debido a la contribución de dosis a los pacientes ${ }^{8}$. Aunque el beneficio es mayor al riesgo, se hace necesaria la implementación de técnicas que permitan la disminución de la dosis durante la exploración. A pesar del advenimiento de nuevos equipos de TC que han incorporado nuevos tipos de asociación de la adquisición de las imágenes al electrocardiograma (ECG) propio del paciente durante la realización del examen, la mayoría de los barridos actualmente se realizan de las maneras clásicas: el gatillado prospectivo, en que se realizan cortes secuenciales solamente en la fase de interés del ciclo cardiaco, y el gatillado retrospectivo, en que la emisión de rayos $X$ es en forma continua de hélice, generando imágenes en todas las fases del ciclo cardiaco aumentando la dosis de radiación. En este tipo de gatillado puede aplicarse la modulación de corriente, la cual consiste en la modificación del mili-amperaje del tubo de rayos $X$ según las características morfológicas de la zona anatómica a evaluar, lo que provoca que la emisión de rayos $X$ se reduzca ${ }^{9}$. Aparte de este tipo de modulación de dosis, otra técnica utilizada para disminuir la dosis de radiación en AngioTC de coronarias es la modulación asociada al ECG o "ECG Pulsing", en donde la corriente se disminuye en el período sistólico y se imparte la corriente seleccionada por el operador en el momento de la diástole. Otra de las técnicas utilizadas es la del Triggering, o escaneo en modo "step and shoot" donde la fase de angiografía se obtiene irradiando de manera secuencial exclusivamente 
durante la diástole, generando una disminución de la dosis lo que hace imposible el análisis de otra fase que no sea la adquirida ${ }^{10,11}$. Actualmente la AngioTC coronaria es realizada con un protocolo de energía del haz de rayos $X$ de 120 kilovolts (kV) y se ha demostrado que la dosis efectiva estimada se asocia a un valor medio de $12 \mathrm{mSv}^{12}$. Hausleiter y cols ${ }^{12}$, en su estudio denominado PROTECTION II, demostraron que una disminución de la energía del haz de 120 a $100 \mathrm{kV}$, no afecta significativamente la calidad de imagen al aplicarlo en pacientes no obesos, siendo éste el principal método de disminución de dosis. Este estudio fue realizado en 400 pacientes, donde 202 fueron asignados al azar a un protocolo que usaba $100 \mathrm{kV}$ y 198 a un protocolo de $120 \mathrm{kV}$, con el objetivo de demostrar la mantención de la calidad de imagen al variar la energía mediante una evaluación que calificaba de 1 a 4 puntos, siendo 1 no diagnóstica y 4 de excelente calidad de imagen y a su vez incluyendo también aspectos técnicos de calidad de imagen. Este estudio comprobó que se reduce la exposición de radiación en un 31\% comparado con el protocolo estándar de $120 \mathrm{kV}$, mientras que al evaluar la calidad de imagen se demostró que la puntuación media fue de $3.3 \pm 0.67$ usando el protocolo de 100 $\mathrm{kV}$, en cambio, con $120 \mathrm{kV}$ fue de $3.28 \pm 0.68$, esta puntuación fue obtenida mediante la apreciación de dos radiólogos expertos en AngioTC coronaria con la escala ya mencionada lo cual demuestra que no se ve afectado debido a que la variación fue solamente de 0,001, valor que no baja del margen de no inferioridad predefinido en $0.2^{12}$.

Existen diversos estudios que se han abocado a la posibilidad de obtener la puntuación de calcio coronario por el método de Agatston con una energía de tubo de $100 \mathrm{kV}$, disminuyendo así la dosis, que concluyen que a pesar de que la disminución del kV informa una óptima correlación en la puntuación realizada con 100 y $120 \mathrm{kV}$, esto implica modificaciones en el análisis estándar, con un aumento en el umbral para la detección de calcio, debido al aumento en la absorción de los rayos $\mathrm{X}$ y el posterior aumento en la atenuación de las placas cálcicas, provocando como consecuencia una sobre estimación de ésta, de tal manera es que la Sociedad de Tomografía Computarizada Cardiovascular no recomienda actualmente el uso rutinario de la exploración de calcio con 100 $\mathrm{kV}$ debido a las modificaciones necesarias para el análisis de la imagen y la escasez de datos clínicos disponibles ${ }^{3,13}$.

La disminución del kV en AngioTC coronaria es una práctica que no se ha integrado en los protocolos de los diversos centros diagnósticos, esto puede deberse a una falta de conocimiento de la estrategia, el desconocimiento de datos científicos que acrediten que se obtiene una imagen diagnóstica o la falta de estandarización de estrategias de disminución de dosis, es por esto que el objetivo del presente estudio fue demostrar la factibilidad de esta estrategia mediante la cuantificación de la disminución de la dosis de radiación y una evaluación de la calidad de imagen obtenida al utilizar un protocolo de adquisición con una energía de tubo de $100 \mathrm{kV}$.

\section{Materiales y métodos}

Se diseñó un estudio comparativo de dos cohortes de pacientes, en que se analizaron de manera retrospectiva exámenes que utilizaron el protocolo habitual de adquisición con $120 \mathrm{kV}$ y luego de manera prospectiva, se aplicó un protocolo modificado de $100 \mathrm{kV}$ para posteriormente comparar las variables dosimétricas y de calidad de imagen entre ambos grupos.

Se incluyeron aquellos pacientes que presentaron un índice de masa corporal (IMC) entre 18,5 y $29,9 \mathrm{~kg} / \mathrm{m}^{2}$, excluyendo pacientes con valores de IMC superiores e inferiores a éstos. Esto se debe a que el aumento de la masa corporal implica una disminución en la dosis de radiación absorbida, por lo que los parámetros técnicos utilizados deben ser aumentados para obtener una imagen diagnóstica, no logrando la disminución de energía. En lo que respecta al límite inferior se ha propuesto en distintos estudios que es posible utilizar una energía del haz de rayos $\mathrm{X}$ de $80 \mathrm{kV}$ para los pacientes bajo este $\mathrm{IMC}^{3}$. Aquellos pacientes que presenten una enfermedad coronaria declarada y tengan estudios anteriores también se excluyeron del estudio.

En el caso de los pacientes retrospectivos los datos ya mencionados se obtuvieron de la ficha clínica del paciente. Se obtuvo el peso en kilogramos y la estatura en metros, para así calcular el IMC a través de la fórmula estándar ${ }^{14}$.

Se obtuvo autorización de la dirección del servicio y del comité de ética local. También se solicitó consentimiento informado firmado por los pacientes para la muestra prospectiva de $100 \mathrm{kV}$.

\section{Adquisición de imágenes}

Las imágenes se obtuvieron de dos equipos multicanal, un TC Siemens ${ }^{\mathrm{TM}}$ Somatom Definition de 64 canales y un General Electric ${ }^{\top \mathrm{M}}$ modelo Optima CT 660 de 64 canales. Para ambos protocolos de energía, se utilizó un protocolo de gatillado electrocardiográfico retrospectivo y modulación de corriente habitual y basada en ECG. Las imágenes adquiridas en Siemens ${ }^{\mathrm{TM}}$ fueron de cortes de 0,6 $\mathrm{mm}$ y un filtro de reconstrucción b26f, mientras que en General Electric ${ }^{\mathrm{TM}}$ el espesor de corte fue de 0,625 $\mathrm{mm}$ con filtro de reconstrucción Soft. Volumen de administración de medio de contraste endovenoso y administración de beta bloqueadores fueron según las indicaciones y decisiones profesionales del servicio local sin intervención alguna de los investigadores. 


\section{Comparación de la calidad de imagen}

Para ambos grupos, la calidad de imagen fue evaluada de manera cuantitativa y cualitativa. Cuantitativamente se analizaron los parámetros técnicos de calidad de imagen de intensidad de señal, ruido, relación señal ruido (SNR) y relación contraste ruido (CNR). Las mediciones en las imágenes se realizaron en el software de visualización de imágenes DICOM, Osirix ${ }^{\mathrm{T}, 15}$. Las mediciones se realizaron en imágenes axiales reconstruidas en un grosor de corte normalizado equivalente a $1 \mathrm{~mm}$ con una región de interés ovalada (ROI) no mayor a $7 \mathrm{~mm}^{2}$. La intensidad de la señal se deriva de la media del número TC (unidades de Hounsfield) promediados de dos ROl's, ubicados en las regiones proximales del lumen de la arteria coronaria izquierda $(\mathrm{ACl})$ y la arteria coronaria derecha (ACD). El ruido fue representado por la desviación estándar del promedio de las unidades de Hounsfield en los mismos ROl's mencionados. La SNR se calculó como la media de los valores de las unidades de Hounsfield medidas en las $A C l$ y $A C D$ y se dividió por el valor del ruido de la imagen. Por último, la CNR se obtuvo de la diferencia de los valores de número Hounsfield de las $A C l$ y $A C D$ en su parte proximal con la densidad media de la pared lateral del ventrículo izquierdo (VI), dividido por el ruido de la imagen ${ }^{12,16}$. En las Figuras $1 a, 1 b$ y $1 c$ se muestra la posición del ROI en cada una de las estructuras medidas.

Cualitativamente se evaluó mediante una puntuación asignada por médicos radiólogos expertos en el área, quienes evaluaron a través de una escala Likert con puntuación de 1 a 4 según los aspectos mencionados en la Tabla 1, considerando la definición de los vasos, el aspecto granuloso de la imagen, artefacto de movimiento, pérdida de contraste y presencia de extensas calcificaciones. Esto se realizó mediante la entrega de una encuesta donde se evaluó la calidad de imagen en la arteria coronaria izquierda, la arteria coronaria derecha, la arteria circunfleja (ACX) y en la arteria descendiente anterior (ADA), donde la evaluación ideal debiese ser de 16 puntos en total, con un esperado de 13 puntos $^{12}$. A los radiólogos evaluadores no se les dio a conocer los parámetros técnicos utilizados en la adquisición de las imágenes y se compararon mediante el promedio de los valores obtenidos para lograr una evaluación entre los protocolos de 120 kV y 100 kV.

\section{Comparación de valores dosimétricos}

Se evalúo en forma comparativa para ambos protocolos el Índice de dosis volumétrico en TC (CTDlvol), producto dosis largo (DLP) y dosis de radiación efectiva estimada. El CTDlvol y DLP se obtuvo para la muestra prospectiva por el informe de dosis o protocolo del paciente entregado por el equipo para cada paciente. Los datos dosimétricos para la muestra retrospectiva se obtuvieron ingresando al cabezal digital de la imagen o meta-data en el cual se buscó los valores de corriente y CTDIvol, mientras que el DLP se logró mediante el producto simple entre CTDI y el largo del barrido. La dosis efectiva se estimó multiplicando el DLP por el factor de conversión correspondiente a la región anatómica irradiada, la cual en este caso es el tórax, cuyo factor es $0,014 \mathrm{mSv}^{*} \mathrm{mGy}-1^{*} \mathrm{~cm}-1$. Se calculó la media aritmética de cada uno de estos factores y se comparó la disminución existente entre ambos protocolos ${ }^{12}$.

Para visualizar si los datos cumplían con una distribución normal y si sus varianzas eran estadísticamente significativas se realizaron las pruebas Kolmogorov - Smirnov. Posteriormente se aplicó la prueba t de Student para la evaluación cuantitativa y la prueba $U$ de Mann Whitney para la evaluación cualitativa con el objetivo de evaluar el grado de significancia en las diferencias de las evaluaciones en ambos protocolos ${ }^{17}$.

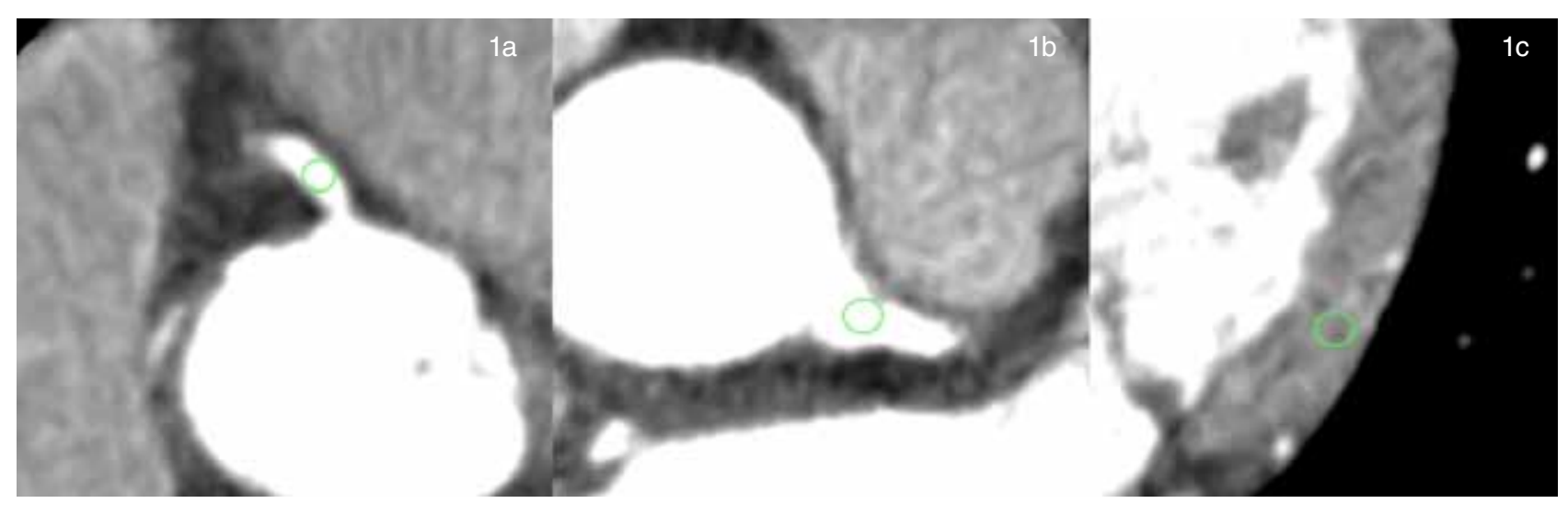

Figura 1. Medición de ROl's. Lugares de medición de UH y desviación estándar en las distintas estructuras cardiacas para la cuantificación de niveles de ruido, intensidad de señal, SNR y CNR. 1a. ROI en origen arteria coronaria derecha. 1b. ROI en origen de arteria coronaria izquierda. 1c. ROI en pared lateral del ventrículo izquierdo. 
Tabla 1. Puntuación para evaluación por médicos radiólogos. Fuente: Hausleiter y cols c,12 $^{8}$

\begin{tabular}{|l|l|l|}
\hline Puntuación & Aspecto de la imagen & Especificación \\
\hline $\mathbf{1}$ & No diagnóstica & $\begin{array}{l}\text { Existe una pérdida de la capacidad de evaluar las arterias } \\
\text { coronarias debido a una extensa pérdida de contorno, artefacto } \\
\text { de movimiento, pérdida de contraste, presencia de extensas } \\
\text { calcificaciones y ruido excesivo en la imagen. }\end{array}$ \\
$\mathbf{3}$ & Adecuada & $\begin{array}{l}\text { Suficiente para descartar una estenosis significativa, pero existe } \\
\text { una mediana pérdida de contorno, artefacto de movimiento, ruido } \\
\text { y disminución del contraste. } \\
\text { Capacidad para evaluar la presencia de estenosis luminal, de placa } \\
\text { aterosclerótica coronaria calcificada y no calcificada, pero existe } \\
\text { una ligera pérdida de contorno, artefacto de movimiento, ruido, } \\
\text { calcificación o bajo contraste. } \\
\text { Capacidad completa de evaluar lumen y estenosis y ausencia de } \\
\text { artefacto de movimiento. }\end{array}$ \\
\hline
\end{tabular}

\section{Resultados}

El estudio fue aplicado a 37 pacientes, de los cuales 20 fueron pacientes que ya se habían realizado una exploración de AngioTC coronaria en la que se empleó la técnica estándar con energía del tubo de $120 \mathrm{kV}$ y los 17 restantes se seleccionaron de aquellos pacientes con indicación de AngioTC y que cumplían con los criterios de inclusión para este estudio. Estos 17 pacientes fueron explorados con el protocolo propuesto de disminución de dosis de $100 \mathrm{kV}$ sin otra modificación de factores técnicos. En la Tabla 2 se pueden observar en detalle las características de los pacientes incluidos en la muestra.

La Tabla 3 muestra los resultados del análisis de calidad de imagen y de dosis de radiación. Respecto del análisis cuantitativo, con el protocolo de 100 kV se observó un aumento significativo en la intensidad de señal de un $26,6 \%(p=0,01)$ y en el ruido un $37,42 \%(p=0,02)$, por otro lado la SNR tuvo un aumento de un 22,6\% ( $p=0,2)$. En cambio, en la CNR se observó una disminución de un 21,17\% $(p=0,2)$. En relación a los resultados de los valores de dosis de radiación con ambos protocolos, el promedio del CTDIvol es significativamente inferior para el protocolo de $100 \mathrm{kV}$ con 19,87 $\pm 5,02 \mathrm{mGy}$ versus el de $120 \mathrm{kV}$ con 40,99 mGy \pm 7,07 mGy $(p<0,001)$, equivalente a un $51,52 \%$. De igual forma el DLP disminuye en un $41,99 \%(p=0,02)$. La dosis efectiva estimada disminuye en un $41,95 \%(p<0,001)$.

Las Figuras 2, 3 y 4 muestran imágenes de exámenes obtenidos con ambos protocolos en las que se pueden observar cualitativamente la mayor atenuación lograda en las estructuras con medio de contraste resultante al utilizar el protocolo de menor energía de tubo.

Tabla 2. Resumen de datos de pacientes.

\begin{tabular}{|c|c|c|}
\hline Características & Protocolo $120 \mathrm{kV}(\mathrm{n}=20)$ & Protocolo $100 \mathrm{kV}(\mathrm{n}=17)$ \\
\hline $\begin{array}{l}\text { Sexo } \\
\text { Masculino } \\
\text { Femenino }\end{array}$ & $\begin{array}{l}6 \\
4\end{array}$ & $\begin{array}{c}12 \\
5\end{array}$ \\
\hline $\begin{array}{l}\text { Peso }(\mathrm{kg}) \\
\text { Altura }(\mathrm{m}) \\
\text { IMC }\left(\mathrm{Kg} / \mathrm{m}^{2}\right)\end{array}$ & $\begin{array}{c}77,1 \pm 11,69 \\
1,71 \pm 0,08 \\
25,91 \pm 2,42\end{array}$ & $\begin{array}{c}74,24 \pm 12,29 \\
1,68 \pm 0,08 \\
25,94 \pm 2,25\end{array}$ \\
\hline $\begin{array}{l}\text { Uso de Beta bloqueadores } \\
\qquad \mathrm{Si} \\
\text { No } \\
\text { Frecuencia cardiaca (lpm) } \\
\text { Largo de barrido }(\mathrm{cm})\end{array}$ & $\begin{array}{c}10 \\
10 \\
59,46 \pm 5 \\
14,43 \pm 2,73\end{array}$ & $\begin{array}{c}7 \\
10 \\
59 \pm 7,9 \\
14,96 \pm 2,04\end{array}$ \\
\hline
\end{tabular}


Tabla 3. Resultados obtenidos de la evaluación de calidad de imagen y dosis de radiación de ambos protocolos.

\begin{tabular}{|c|c|c|c|}
\hline Parámetro estudiado & $\begin{array}{l}\text { Protocolo } 120 \mathrm{kV} \\
(\mathrm{n}=20)\end{array}$ & $\begin{array}{l}\text { Protocolo } 100 \mathrm{kV} \\
(n=17)\end{array}$ & p-Valor \\
\hline $\begin{array}{l}\text { Evaluación cualitativa* } \\
\text { Calidad de imagen global }\end{array}$ & $3,25 \pm 0,8$ & $3,41 \pm 0,7$ & 0,58 \\
\hline $\begin{array}{l}\text { Score por arteria }{ }^{*} \\
\text { Arteria coronaria izquierda } \\
\text { Arteria descendiente anterior } \\
\text { Arteria circunfleja } \\
\text { Arteria coronaria derecha }\end{array}$ & $\begin{array}{c}3,4 \pm 0,8 \\
3,25 \pm 0,9 \\
3,3 \pm 0,9 \\
3,15 \pm 0,81\end{array}$ & $\begin{array}{c}3,36 \pm 0,8 \\
3,47 \pm 0,79 \\
3,4 \pm 0,8 \\
3,29 \pm 0,91\end{array}$ & $\begin{array}{l}0,77 \\
0,6 \\
0,5 \\
0,4\end{array}$ \\
\hline $\begin{array}{l}\text { Evaluación cuantitativa }{ }^{\star \star} \\
\text { Intensidad de señal (prom, UH) } \\
\text { Ruido }(\%, \text { UH) } \\
\text { Relación Señal/Ruido SNR } \\
\text { Relación Contraste/Ruido CNR }\end{array}$ & $\begin{array}{c}372,70 \pm 44,67 \\
35,91 \pm 9,42 \\
22,28 \pm 6,95 \\
19,50 \pm 6,14\end{array}$ & $\begin{array}{c}472,03 \pm 78,61 \\
57,39 \pm 14,22 \\
17,24 \pm 4,76 \\
15,37 \pm 4,45\end{array}$ & $\begin{array}{c}0,01 \\
0,02 \\
0,2 \\
0,2\end{array}$ \\
\hline $\begin{array}{l}\text { Dosis**} \\
\text { CTDI }{ }_{\text {vol }}(\mathrm{mGy}) \\
\text { DLP (mGy*cm) } \\
\text { Dosis efectiva estimada (mSv) }\end{array}$ & $\begin{array}{c}40,99 \pm 7,07 \\
599,36 \pm 186,92 \\
8,39 \pm 2,61\end{array}$ & $\begin{array}{c}19,87 \pm 5,02 \\
347,68 \pm 122,67 \\
4,87 \pm 1,71\end{array}$ & $\begin{array}{c}<0,001 \\
0,002 \\
<0,001\end{array}$ \\
\hline
\end{tabular}
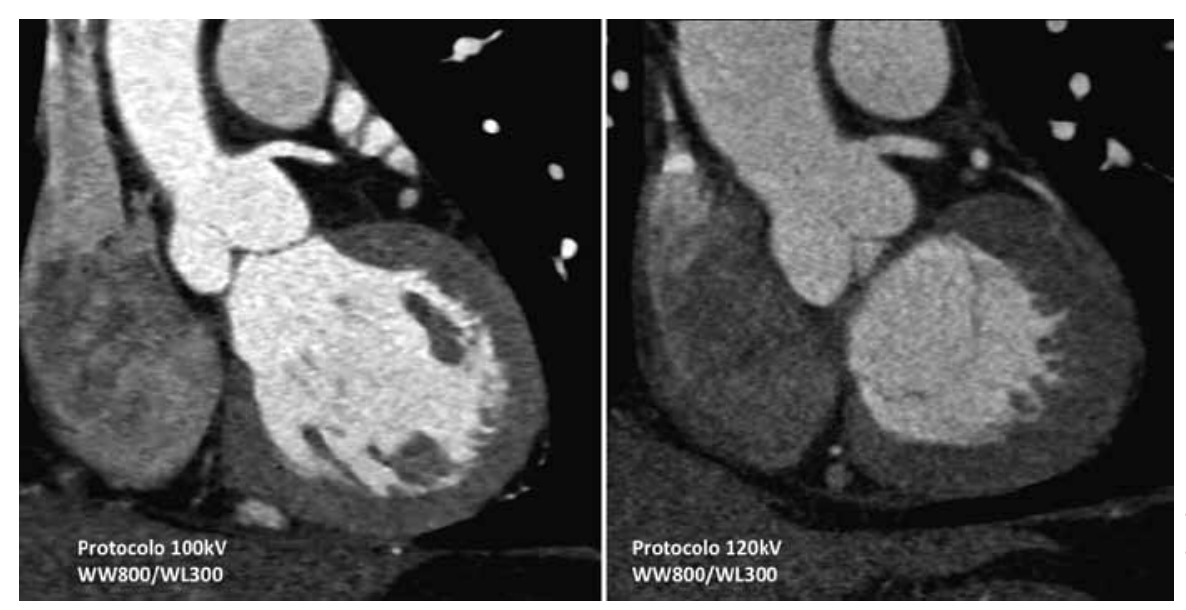

Figura 2. Reformateos coronales de exámenes adquiridos con ambos protocolos. Se usaron los mismos parámetros de ancho y nivel de ventana.
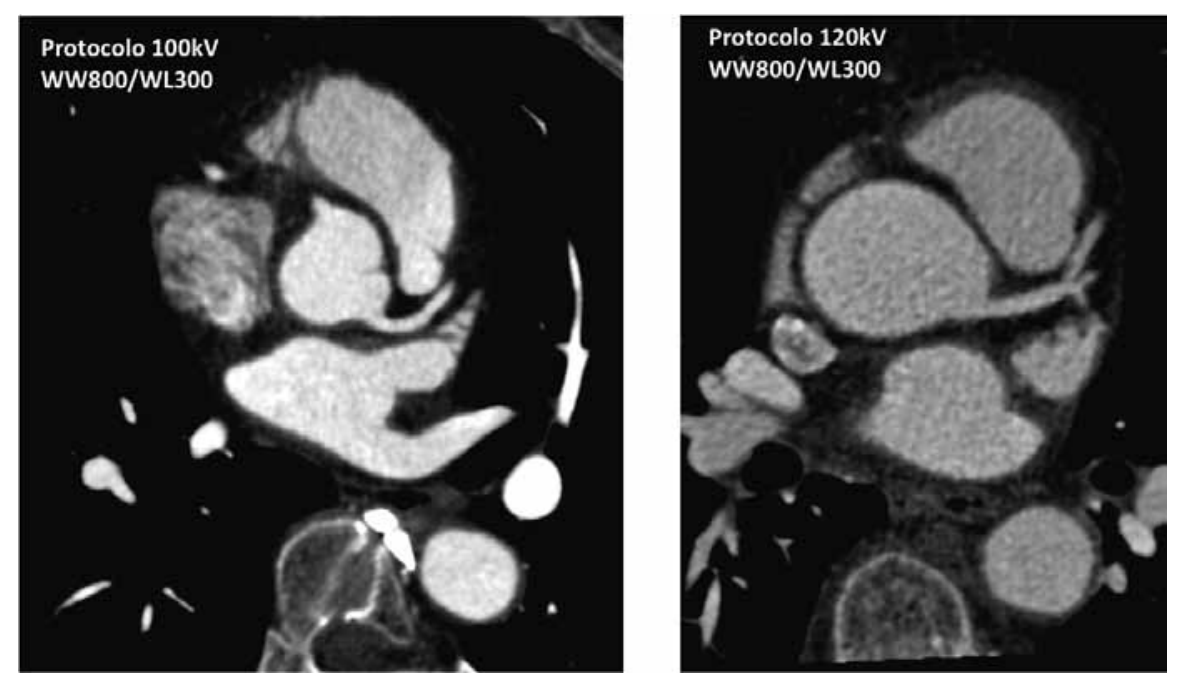

Figura 3. Cortes axiales de dos exámenes adquiridos con ambos protocolos. Se usaron los mismos parámetros de ancho y nivel de ventana. 

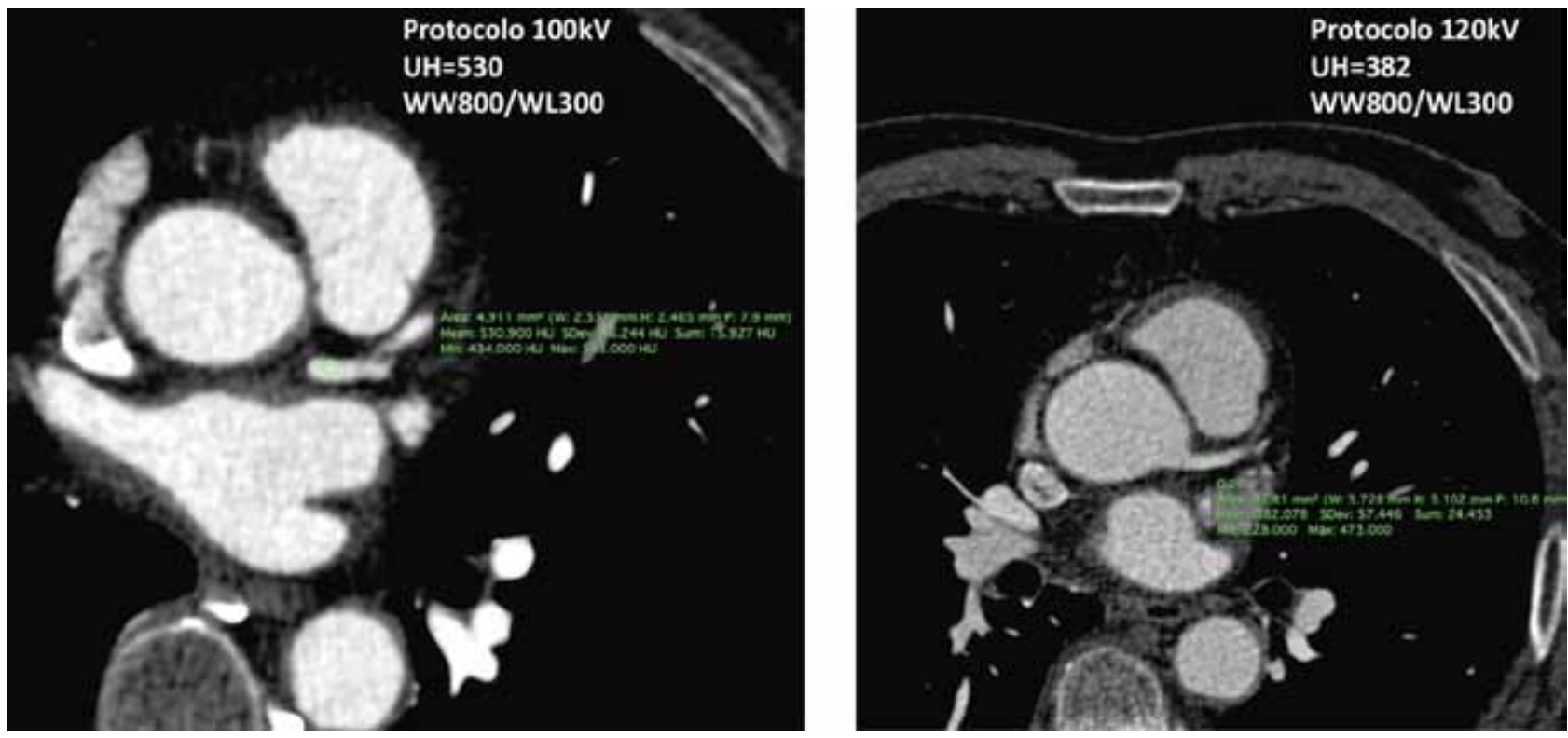

Figura 4. Intensidad de señal en los orígenes de la arteria coronaria izquierda en exámenes obtenidos con los dos protocolos. Se usaron los mismos parámetros de ancho y nivel de ventana.

La Figura 5 muestra una imagen de exámenes obtenidos con ambos protocolos en la que se puede observar el mayor nivel de ruido (como desviación estándar de UH) producto de la disminución de la energía del tubo.

En el análisis cualitativo al comparar ambos protocolos se evidencia un aumento de un $4,9 \%(p=0,58)$ en la puntuación total de la encuesta realizada por los radiólogos al utilizar el protocolo de $100 \mathrm{kV}$. Las puntuaciones promedio de cada una de las arterias se pueden apreciar en la Tabla 3. Se presentaron 4 evaluaciones con puntuaciones inferiores a 3 en aquellas imágenes obtenidas con un protocolo de $100 \mathrm{kV}$, equivalentes al $23,5 \%$ del total. Estas fueron a causa de artefacto de movimiento $(50 \%)$ y a causa del ruido $(50 \%)$. Mientras que en aquellas obtenidas con 120 $\mathrm{kV}$ aquellas evaluaciones inferiores a $3(20 \%)$ fueron a causa de artefactos de movimiento y baja densidad.
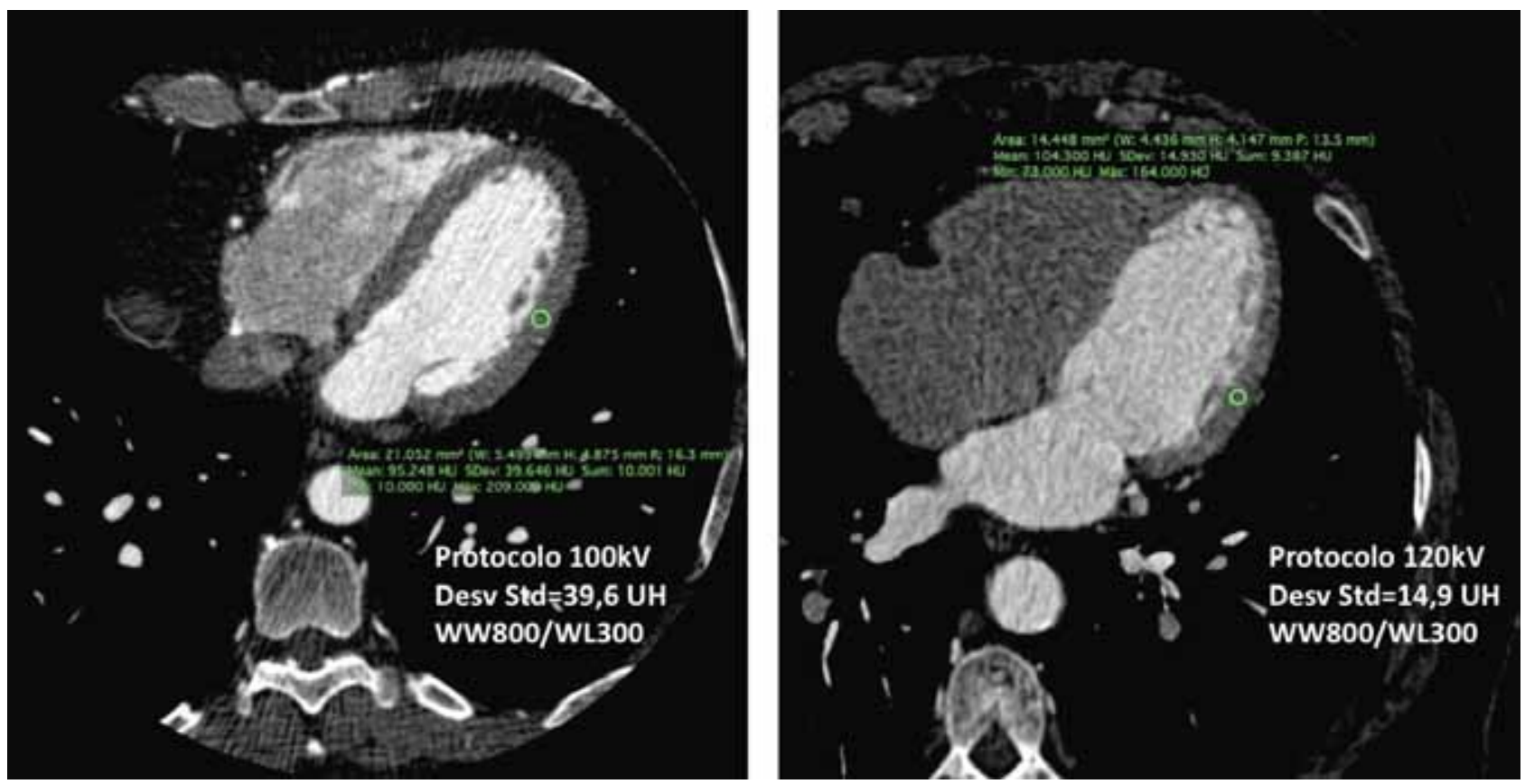

Figura 5. Nivel de ruido (desviación estándar, UH) medido en la pared lateral del ventrículo izquierdo en dos exámenes obtenidos con los diferentes protocolos. 


\section{Discusión y conclusiones}

La disminución de dosis junto a la protección radiológica al paciente han sido una preocupación e interés permanente de los profesionales encargados de realizar los exámenes radiológicos. A pesar de que aún existe controversia sobre la relación de los riesgos estocásticos de la radiación recibida por exámenes imagenológicos, el principio ALARA, que indica que la dosis de radiación a un paciente debe ser tan baja como razonablemente sea posible, es bien aceptado. Esto implica que la disminución de dosis entregada debe ser la máxima posible sin afectar la calidad diagnóstica de la imagen ni la relación riesgo-beneficio del paciente, un estudio no diagnóstico puede implicar la realización de imágenes adicionales aumentando la radiación neta entregada al paciente ${ }^{18}$.

En el presente estudio se evaluaron las diferencias en la calidad de imagen entre los protocolos de $120 \mathrm{y}$ $100 \mathrm{kV}$ de AngioTC coronaria realizada en pacientes no obesos y sin enfermedad coronaria declarada, y se demostró que la calidad de imagen es similar y superior, aunque con una variación no significativa $(p=0,58)$ al usar el protocolo de $100 \mathrm{kV}$, manteniéndose el nivel de confianza diagnóstica con una dosis de radiación estimada inferior. La disminución de kilo-voltaje implicó una elevación en la intensidad de señal y ruido. El aumento de la intensidad de señal se explica por las características de absorción de los rayos $\mathrm{X}$ del yodo, el cual es superior a energías de menor $\mathrm{kV}$, traduciéndose en una mayor atenuación del medio de contraste. Mientras que el aumento de ruido se explica por la reducción de kV en los parámetros de adquisición. La disminución del voltaje del tubo se ha demostrado como una estrategia efectiva de optimización de dosis de radiación en adultos de talla baja mejorando además la visualización de estructuras óseas u ocupadas con medio de contraste debido a un aumento en su atenuación ${ }^{21}$, incluso con el uso de $80 \mathrm{kV}$ exclusivamente en pacientes de talla pequeña ${ }^{22}$.

Un estudio multicéntrico en que se realizó la comparación de imágenes con la modificación de energía en AngioTC coronaria en 400 pacientes no obesos, por Hausleiter y col, del cual derivaron múltiples estudios pequeños con el mismo fin, se demostró que se mantiene la calidad de imagen al utilizar un protocolo de $100 \mathrm{kV}$ disminuyendo en un $31 \%$ la dosis de radiación, desde un promedio de DLP de $868 \mathrm{mGy}^{*} \mathrm{~cm}$ a uno de $599 \mathrm{mGy}^{*} \mathrm{~cm}$, equivalente a una reducción de dosis efectiva de 12,2 mSv a 8,4 mSv. Esto implicó un aumento en el ruido de un $36 \%$ desde una media de 24,6 a 33,5 UH, lo cual solamente afecta la imagen de manera estética ya que no impidió la evaluación diagnóstica de las arterias coronarias por los radiólogos evaluadores ${ }^{12}$. En el presente estudio, el promedio de DLP disminuyó en un $41,99 \%$ desde un promedio de 599,36 $\mathrm{mGy}^{*} \mathrm{~cm}$ a $347,68 \mathrm{mGy}^{*} \mathrm{~cm}$, equivalente a una reducción de dosis efectiva estimada desde $8,39 \mathrm{mSv}$ a $4,87 \mathrm{mSv}$, con un aumento en el ruido de un $37 \%$. Se logró evidenciar que la dosis efectiva para el protocolo de $120 \mathrm{kV}$ utilizado en este estudio, es cercana a la entregada utilizando $100 \mathrm{kV}$ en la ACTC del estudio de Hausleiter. A pesar de utilizar la misma energía del haz de rayos $X$ las diferencias presentadas entre los estudios en las dosis efectivas estimadas y CTDIvol se pueden explicar debido a las diferencias en el mili amperaje utilizado en ambos estudios, siendo menor el utilizado en la presente investigación.

La modalidad gold standard para el estudio de las arterias coronarias, continúa siendo la angiografía convencional intervencionista o coronariografía, con dosis efectivas reportadas entre 7 - $30 \mathrm{mSv}$ dependiendo de si es diagnóstica o terapéutica, aunque la dosis mínima entregada por ésta es levemente menor a la entregada por el protocolo actual de la AngioTC coronaria a $120 \mathrm{kV}$, entonces una reducción de la energía del haz de rayos $\mathrm{X}$ a $100 \mathrm{kV}$ representa una disminución de dosis entregada al paciente de 30,57\% en relación a la angiografía convencional, por lo que debe considerarse para disminuir el número de intervenciones en pabellón innecesarias. También debe compararse la dosis con otros estudios cardiacos habituales de medicina nuclear como la perfusión miocárdica con Tecnecio 99m-Sestamibi o Talio en estrés y reposo, que implican $9 \mathrm{mSv}$ y $41 \mathrm{mSv}$ respectivamente ${ }^{19,20}$.

En la evaluación cualitativa realizada por los radiólogos, se obtuvo un promedio de 3,25 vs uno de 3,41 para los protocolos de 120 y 100 kV respectivamente, junto a una puntuación total de 13,27 levemente por sobre el esperado de 13, obteniendo entonces una calidad de imagen capaz de ser interpretada en forma diagnóstica por los radiólogos. Se presentaron puntuaciones 1 y 2 en alguna de las arterias evaluadas con el protocolo de baja energía, en sólo cuatro ocasiones, debidas a artefactos de movimiento en un $50 \%$ y a causa del aumento de ruido de la imagen en el mismo porcentaje, estas últimas coincidían con aquellos pacientes que se encontraban muy cercanos al IMC tope de inclusión al estudio, pacientes que se encontraban entre un IMC de 28 y $29 \mathrm{~kg} / \mathrm{m}^{2}$. Lo anterior hace tomar en cuenta, en aquellos pacientes con IMC más altos como los mencionados, la distribución de la masa corporal del paciente para decidir el protocolo a utilizar ${ }^{3}$.

Una optimización de dosis de radiación en AngioTC coronario no deber ser sólo a causa de una disminución de energía de tubo, sino que debe ser producto de la utilización en conjunto de las distintas estrategias y aplicaciones existentes de los tomógrafos, como el gatillado prospectivo, la modulación de corriente habitual y la basada en ECG así como la incorporación de métodos iterativos de reconstrucción ${ }^{3,12}$. Si bien 
la dosis es un buen determinante del resultado final de la imagen en la AngioTC Coronaria, la calidad de imagen depende de un conjunto de factores, en primer lugar una alta calidad diagnóstica es posible de lograr utilizando equipos multidetectores de 64 canales o superiores, ya que permiten una mayor rapidez en el barrido consiguiendo una apnea en forma efectiva, una alta resolución espacial que permite evaluar nítidamente estructuras finas como las arterias coronarias y una alta resolución temporal permitiendo adquirir el corazón en diástole, momento en que el corazón presenta menor movimiento. Además, una frecuencia cardiaca baja y estable permite un periodo diastólico largo para alcanzar a adquirir imágenes sin artefactos de movimiento. El cumplir con las condiciones anteriormente mencionadas es parte también de evitar una entrega de dosis mayor a la necesaria por requerir de más exploraciones para conseguir la imagen diagnóstica deseada ${ }^{19}$.

Como limitación del presente estudio se encuentra el tamaño pequeño de la muestra, siendo ésta representativa sólo del centro estudiado. Datos más representativos y numerosos podrían ser adquiridos si se realizara un estudio multicéntrico y de distintas marcas de equipos, en centros clínicos donde se realicen AngioTC coronaria a nivel nacional. Por otro lado, en este estudio sólo se evaluó la disminución de dosis de la fase angiográfica del examen de Angio TC coronaria y no se consideró la dosis entregada por la imagen localizadora, la exploración para la puntuación de calcio, ni la entregada por la exploración del bolus tracking, que implica un estudio completo, sin embargo, la dosis de radiación significativa del examen es producto de la fase angiográfica, que fue la estudiada en la presente investigación.

Como proyección se espera entregar los resultados dosimétricos y de los parámetros que respectan a la calidad de imagen con el fin de lograr una futura implementación del protocolo de $100 \mathrm{kV}$ en el servicio de radiología de la institución, para realizar la AngioTC coronaria en aquellos pacientes que cumplan con los criterios definidos en el estudio.

Una de las grandes preocupaciones en el área de los exámenes diagnósticos en radiología es el uso de radiación ionizante debido a los posibles riesgos que esto trae consigo, es por ello la preocupación de la optimización de dosis, como la realizada en este estudio. Una reciente revisión de las principales conclusiones de la Conferencia Iberoamericana de Protección Radiológica en Medicina ${ }^{23}$, señala que el principal problema en radiología diagnóstica es la falta de cultura de radioprotección del paciente, con implicancias en justificación de los estudios de imágenes y una pobre optimización de dosis de radiación en los procedimientos, por lo que es importante hacer énfasis en la educación al respecto y con un rol muy importante de las sociedades de profesionales, en particular tecnólogos médicos y radiólogos, tema al que fue dirigido el presente estudio.

Como conclusión se puede señalar que la utilización de un protocolo de AngioTC coronaria con 100 kV permite disminuir la dosis de radiación entregada al paciente sin perjudicar cualitativamente la calidad de imagen, en los pacientes seleccionados del presente estudio. A pesar del aumento del ruido en la imagen, éste no impide la evaluación diagnóstica por los médicos radiólogos. Por lo cual se sugiere considerar e incorporar este protocolo con disminución de energía de tubo para pacientes no obesos y sin enfermedad coronaria declarada para así entregar una dosis de radiación tan baja como razonablemente sea posible.

Este estudio representa sólo la disminución de dosis en la AngioTC coronaria, por lo que futuras investigaciones pueden considerar esta estrategia de disminuir la energía del tubo para el resto de las angiografías realizadas por TC a distintas zonas anatómicas.

\section{Agradecimientos}

Los autores desean agradecer a los tecnólogos médicos, radiólogos y dirección del servicio de diagnóstico por imágenes por la disposición a la presente investigación.

\section{Referencias}

1. Organización Mundial de la Salud, OMS. Las 10 causas principales de defunción en el mundo [Internet]. World Health Organization; [cited 2015 Apr 27]. Disponible en: http://www.who.int/mediacentre/factsheets/fs310/ es/index2.html

2. Departamento de Estadísticas e Información de Salud, DEIS, MINSAL. INDICADORES BÁSICOS DE SALUD. Santiago, Chile; 2013.

3. Halliburton SS, Abbara S, Chen MY, Gentr R, Mahesh, M, Raff GL, et al. SCCT guidelines on radiation dose and dose-optimization strategies in cardiovascular CT. Journal of cardiovascular computed tomography 2011; 5(4): 198-224. doi:10.1016/j.jcct.2011.06.001

4. Smith-Bindman R, Lipson J, Marcus R, Kim KP, Mahesh $M$, Gould et al Radiation dose associated with common computed tomography examinations and the associated lifetime attributable risk of cancer. Archives of internal medicine 2009; 169(22): 2078-2086.

5. Raff G, Chinnaiyan K. Papel del angio-TAC coronario en la clasificación precoz de los pacientes con dolor torácico agudo. Rev Esp Cardiol 2014; 62(9): 961-965.

6. Bushong S. Manual de radiología para técnicos. 9a ed. Barcelona. Elsevier Inc 2010; 449-465.

7. Soto J. Tomografía Computada Cardiaca. Depto Med y Unidad Cardiol Campus Occident Univ Chile Depto Enfermedades Cardiovasc Clínica Las Condes. 2010; 26(1): 9-16.

8. Hausleiter J, Meyer T, Hermann F, Hadamitzky M, Krebs M, Gerber TC, et al. Estimated Radiation Dose Associated With Cardiac CT Angiography. JAMA 2009; 301(5): 500-507.

9. Hausleiter J, Meyer TS, Martuscelli E, Spagnolo P, 
Yamamoto $\mathrm{H}$, Carrascosa $\mathrm{P}$, et al. Image quality and radiation exposure with prospectively ECG-triggered axial scanning for coronary CT angiography: the multicenter, multivendor, randomized PROTECTIONIII study. JACC Cardiovasc Imaging 2012 May; 5(5): 484-493.

10. Jakobs TF, Becker CR, Ohnesorge B, et al. Multislice helical CT of the heart with retrospective ECG gating: reduction of radiation exposure by ECG-controlled tube current modulation. Eur Radiol 2002; 12(5): 1081-1086.

11. Hausleiter J, Meyer T, Hadamitzky M, et al. Radiation dose estimates from cardiac multislice computed tomography in daily practice: impact of different scanning protocols on effective dose estimates. Circulation 2006; 113(10): 1305-1310.

12. Hausleiter J, Martinoff S, Hadamitzky M, Martuscelli E, Pschierer I, Feuchtner GM, et al. Image quality and radiation exposure with a low tube voltage protocol for coronary CT angiography results of the PROTECTION II Trial. JACC Cardiovasc Imaging 2010 Nov; 3(11): 1113-1123.

13. Deprez F, Vlassenbroek A, Ghaye B, Raaijmakers R, Coche E. Controversies about effects of low-kilovoltage MDCT acquisition on Agatston calcium scoring. $J$ Cardiovasc Comput Tomogr 2013; 7(1): 58-61.

14. Moreno M. Definición y Clasificación de la Obesidad. Rev Med Clin Condes 2012; 23(2): 124-128.

15. Rosset A, Spadola L, Ratib O. OsiriX: an open-source software for navigating in multidimensional DICOM images. Journal of digital imaging 2004; 17(3): 205-216.

16. Bischoff B, Hein F, Meyer T, Krebs M, Hadamitzky M, Martinoff S, et al. Comparison of sequential and helical scanning for radiation dose and image quality: results of the Prospective Multicenter Study on Radiation Dose Estimates of Cardiac CT Angiography (PROTECTION) I Study. AJR Am J Roentgenol 2010 Jun; 194(6): 14951499.

17. Freund JE., Simon G. A. Estadística elemental. México: Pearson Educación. 1994

18. Ramos O, Villarreal M. Disminución de la dosis de radiación en el radiodiagnóstico. Rev Chil Radiol 2013; 19(1): 5-11.

19. Bitar P. Evaluación cardiaca con tomografía computada y resonancia magnética. Rev Med Clin Condes 2013; 24(1): 54-62.

20. Gerber T, Jeffrey C, Arai A, Dixon R, Ferrari V, Gomes $A$, et al. lonizing radiation in cardiac imaging: $A$ science advisory from the American Heart Association Committee on cardiac imaging of the council on clinical cardiology and committee on cardiovascular imaging and intervention of the council on cardiovascular radi. Circulation 2009; 119(7): 1056-1065.

21. Castro D. Efectos del voltaje de tubo en la dosis de radiación y calidad de imagen en fantoma en tomografía computarizada multicorte pediátrica. Rev Chil Rad 2016; 22(1): 20-26 http://dx.doi.org/10.1016/j. rchira 2016.02.004

22. Andreini $D$ et al. Coronary CT angiography with 80 $\mathrm{kV}$ tube voltage and low iodine concentration contrast agent in patients with low body weight. Journal of Cardiovascular Computed Tomography 2016; 10: 322-326.

23. Soffia P, Ubeda C, Miranda P, Rodríguez JL. Radioprotección al día en radiología diagnóstica: Conclusiones de la Conferencia Iberoamericana de Protección Radiológica en Medicina (CIPRaM) 2016. Rev Chil Radiol 2017; 23(1): 15-19. 